\title{
Desarrollo de un Dispositivo basado en el Concepto de Enmascarador de Voz
}

\section{Development of a Device Based on the Concept of Enmascarador of Voice}

\author{
Emilio Villavicencio Chamorro, Daniel O. García Romero, Leopoldo Yabar Escribane/(*)
}

\section{RESUMEN}

En nuestro país el tratamiento básico para mejorar o superar el problema de realimentación auditiva debido a la falta de utilización del canal propioceptivo (síndrome de la tartamudez) es la terapia de lenguaje, que se basa en trabajos de ritmo respiratorio, trastornos de fonación y articulación verbal; en algunos de los casos reciben adicionalmente ayuda psicológica. Entre tanto, en los países desarrollados además de los tratamientos mencionados incorporan sofisticados equipos médicos (audiómetros) para que el tratamiento sea mucho más eficaz, por consiguiente un mejoramiento notorio en menos tiempo. En este sentido, el presente trabajo va en búsqueda de tal fin, con el diseño y desarrollo de un dispositivo electrónico portátil de bajo consumo de energía. Este dispositivo emite un sonido (tono) de intensidad y frecuencia variable de tal modo que se ajuste a las características auditivas de cada persona (tono agradable) al momento cuando habla la persona tartamuda, es decir, cuando el micrófono detecte la presencia de voz del sujeto y es transmitido a sus oídos por medio de los auriculares, de modo que el sujeto no hace una realimentación auditiva de su habla sino propioceptiva. Con esta alteración en la realimentación auditiva permite a la persona con disfémia superar los problemas de ansiedad y consecuentemente una mejora en su fluidez del habla.

\begin{abstract}
In our country, the basic treatment to improve or overcome the problem of auditory feedback due to lack of use of the proprioceptive channel (stuttering syndrome) is the speech therapy, which is based on work of breathing, phonation and articulation disorders verbal, in some cases additionally receive psychological help. Meanwhile, in developed countries in addition to the above treatments incorporate sophisticated medical equipment (audiometers) that the treatment is much more effective, therefore a noticeable improvement in less time. In this sense, this work goes in search of purpose, with the design and development of a portable electronic device with low power consumption. This device emits a sound (tone) of variable intensity and frequency in such a way that conforms to the auditory characteristics of each person (nice tone) at the time when the person speaking stuttering, ie, when the microphone detects the presence of voice subject and is transmitted to your ears through headphones, so the subject does not auditory feedback of their speech but proprioceptive. With this altered auditory feedback allows the person with disfémia overcome the problems of anxiety and an improvement in their fluency of speech.
\end{abstract}

\section{INTRODUCCIÓN}

En nuestro país como en cualquier parte del mundo las personas que padecen de alguna discapacidad se encuentran en una situación de desamparo tanto político como social e incluso familiar.

La última encuesta realizada por INEI-ENCO revela que existen cerca de dos millones de peruanos que padecen de alguna discapacidad en donde cerca de 20 mil peruanos tienen algún problema en el habla Fig. 1.

Es visible deducir que la discapacidad en el Perú está fuertemente ligada a la pobreza imponiéndose límites para la vida y el trabajo de aquellas personas. Frente a la privación de oportunidades, aquellas personas migran hacia las ciudades para buscar soluciones a su

(") Grupo de I\&D en Ingeniería Biomédica, Facultad de ingeniería Electrónica, Universidad Tecnológica del Perú 
situación económica y social, en consecuencia solo encuentran desnutrición, desempleo y analfabetismo, condiciones; que provocan otras deficiencias en la población entre ellas nuevas discapacidades, ya sean congénitas o adquiridas (después de nacer). En efecto en la constitución y en algunas leyes y en los planes de desarrollo se proponen medidas para afrontar este problema pero es muy poco lo que se hace, entre tanto en los Pactos Internacionales y las Cumbres donde tienen como resultado casi exclusivo que luego estos compromisos no son asumidos. En este contexto es irrefutable la necesidad de que el Estados y las Instituciones asuman un rol más activo en la generación tanto de profesionales por parte de las instituciones de educación y los canales para que estos profesionales se desenvuelvan en el medio adecuado, por parte del Estado, para la solución de problemas como la discapacidad en los peruanos [1].

La discapacidad en el habla, esta dado por el Síndrome de la Tartamudez (Disfemia). Como sabemos el hombre es un ser desprotegido incapaz de valerse por sí mismo al momento de nacer, es así que pasa gran parte de los primeros años de su infancia tratando de controlar procesos que más adelante le van ayudar a sobrevivir como el ver, el caminar, el hablar, y es este último, proceso en donde se diferencia de las demás especies, y donde invierte gran parte de su vida para controlarlo [2]. Sabiendo que el cuerpo humano es un sistema realimentado en donde el canal de realimentación es pre-determinado por los subsistemas en el aprendizaje y control de las variables en los procesos antes señalados, es decir, para cada proceso en donde se quiera controlar distintas variables hay un específico canal de realimentación hacia el cerebro donde se encuentra el núcleo del sistema nervioso. En el caso del habla el canal de realimentación es el canal propioceptivo, un conjunto de conductos y nervios que conectan la salida de la voz en donde se encuentran su frecuencia fundamental con sus armónicos, hacia el oído medio para acoplarlo hacia el oído interno, en donde el tiempo en ser escuchada la voz por este canal a diferencia de ser escuchado por el oído externo, es donde el cerebro aprovecha en procesar la siguiente palabra a pronunciar, es decir al intentar escuchar por el oído externo en vez de escuchar por el canal propioceptivo ocurre un retarde una desincronización entre el sistema fonatorio y el sistema auditivo. Por consiguiente el presente trabajo va en búsqueda de tal fin, en desarrollar un dispositivo que enmascare la voz con un tono (ruido blanco), sea portátil y de bajo consumo de energía en donde tenga por función la interrupción de una realimentación auditiva de la voz hacia una propioceptiva [3], [4]..

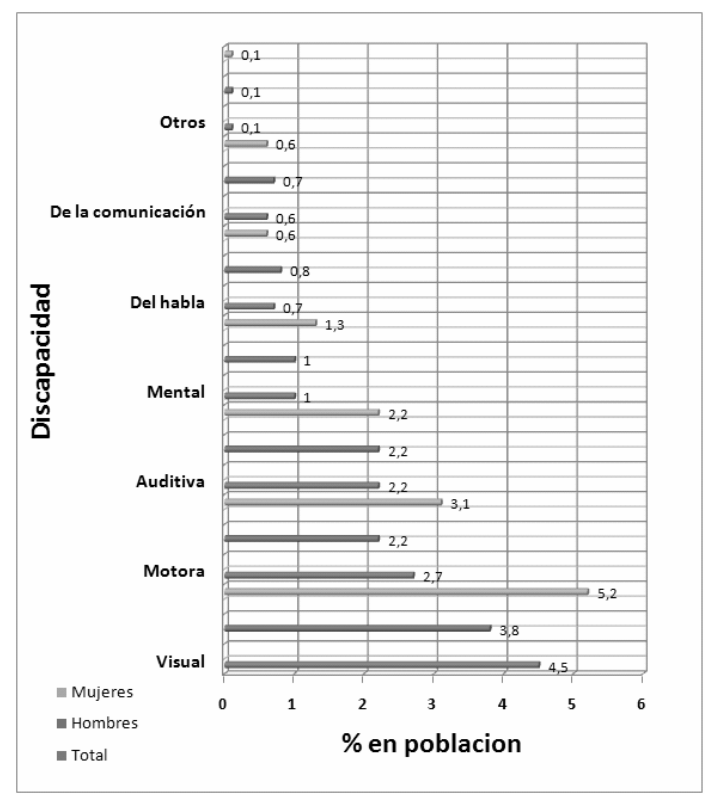

Fig. 1: Estimaciones de población discapacitada en Perú.

\section{METODOLOGÍA}

\section{Marco fisiológico}

Sistema Auditivo, está conformado por el oído externo, medio e interno, los estímulos sonoros pasan por estas zonas para el procesamiento mecánico de las ondas y la conversión a señales electroquímicas [5]. Las frecuencias e intensidades audibles en las personas son $20 \mathrm{~Hz}-20 \mathrm{KHz}$ y $0 \mathrm{db}-180 \mathrm{db}$ respectivamente.

Sistema Fonatorio, está compuesto por tres grupos de órganos: órganos de respiración (pulmones, bronquios y tráquea), órganos de fonación (laringe, cuerdas vocales, cavidades nasal y oral), órganos de articulación (paladar, lengua, dientes, labios). Todos estos órganos tienen que tener una coordinación y precisión en sus movimientos para generar el habla, de tal forma que son controlados por el sistema 
nervioso y supervisados por el sistema auditivo por medio del canal propioceptivo [6].

\section{Técnicas y apoyo tecnológico existentes}

Contracondicionamiento, Esta técnica exige que el sujeto deba ser entrenado para interrumpir su habla en el momento de tartamudear, para inspirar profundamente. [7] - [9].

Entrenamiento con metrónomos, Permite a la persona tartamuda seguir un ritmo al hablar, haciendo coincidir sus sílabas, palabras o frases con el golpeteo regular de un aparato musical llamado metrónomo [9].

Dispositivos enmascarador de voz, son aparatos que impiden al sujeto oír su propia voz, haciendo desaparecer la realimentación auditiva y el mantenimiento de la ansiedad. (Fig. 2) [7] - [9]

\section{Corrección de la realimentación auditiva distorsionada}

La producción del habla envuelve un proceso de realimentación en bucle cerrado, esto significa que el hablante está continuamente monitorizando y chequeando su propia producción de voz. En los tartamudos tienen un defecto en el sistema de monitorización, necesario para producir el habla secuencial y que el problema puede deberse a una realimentación auditiva distorsionada. Una forma de corregirlo es usando sonidos que impide al tartamudo escuchar su propia habla (ensordecerle cada vez que habla) imposibilitándole engolfar en sus propias autoevaluaciones erróneas (corrigiendo la realimentación auditiva distorsionada), y como resultado se logra incrementar y mejorar la fluidez de los tartamudos liberándole además de la ansiedad [5].

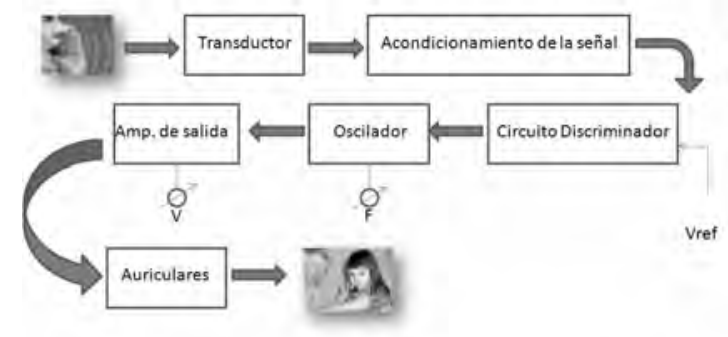

Fig. 2: Diagrama de Bloques del Dispositivo Electrónico de Enmascaramiento.
El sonido generado por el dispositivo presenta una frecuencia variable comprendida dentro del espectro audible $465 \mathrm{~Hz}$ a $2 \mathrm{KHz}$. y con una intensidad muy por debajo de los $80 \mathrm{~dB}$ que son los niveles óptimos para que el tratamiento sea mucho más efectivo [6]

\section{Diagrama de Bloques}

El dispositivo consta de seis (06) etapas donde cada una cumple con funciones determinadas como se observa en la Fig. 2.

La etapa de transductor tiene por objeto captar y convertir las ondas sonoras producida por el individuo a señal eléctrica mediante un transductor que propiamente dicho es un micrófono; además posee un elemento resistivo, que posibilita la obtención de la máxima sensibilidad del micrófono y de un capacitor, que actúa como desacople de la corriente continua de alimentación, ver Fig. 3. El tipo de micrófono empleado es el tipo Electret debido a que posee características similares al micrófono tipo capacitivo, es decir, tiene alta sensibilidad entre 5 y $15 \mathrm{mV} / \mathrm{Pa}$, alta fidelidad (respuesta casi plana de $50 \mathrm{~Hz}$ a $10 \mathrm{Khz}$.) y bajo costo [10]. Los niveles de voltaje que presentan en la salida del micrófono son muy bajos (entre $40 \mathrm{mV}$ a $70 \mathrm{mV}$ ) para ser tratados adecuadamente por la etapa de Acondicionamiento de señal.

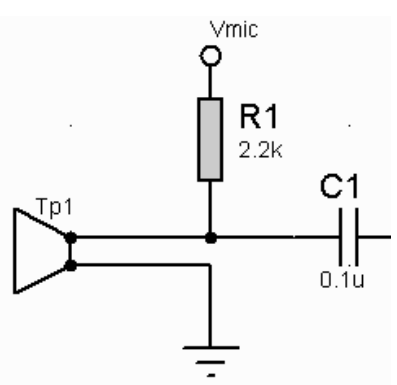

Fig. 3: Etapa Transductor.

La etapa de acondicionamiento de señal consta básicamente de un amplificador inversor, el LF351N de entrada FET, por sus características de alta impedancia de entrada, es apropiado en la adaptación de impedancia con la etapa anterior, y su bajo consumo de energía. Es importante mencionar que la señal de salida de esta etapa en algunas otras siguientes etapas, se encuentra montada sobre una señal 
continua de 4.5 debido a que todos los amplificadores operacionales y otros elementos utilizados son alimentados con una sola batería, ver Fig. 4, satisfaciendo los requerimientos de diseño exigido, pequeño, ligero, cómodo y portátil; por tal motivo se debe suministrar una señal continua a la entrada no inversora del amplificador operacional para que la señal a tratar o amplificar no sea recortada

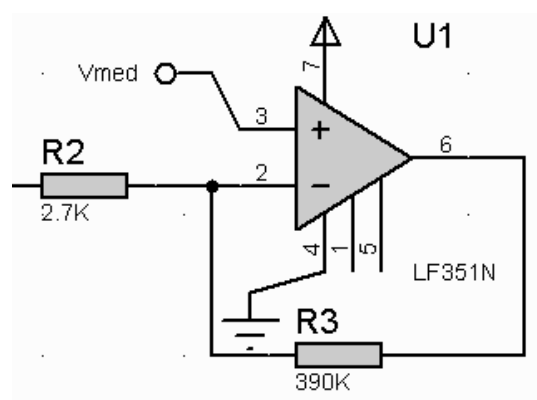

Fig. 4: Etapa Acondicionamiento de la Señal.

Etapa circuito discriminador consiste en determinar si la señal entrante al micrófono pertenece a la voz de la persona que está hablando o al ruido generado por cualquier otra fuente que no sea el habla; en el primer caso el circuito discriminador la dejara pasar para activar la etapa del circuito oscilador, mientras que en el segundo caso será rechazada. Está constituido por dos circuitos, un rectificador de $1 / 2$ onda y un comparador. El primer circuito es un rectificador de precisión ya que es capaz de rectificar la señal completa inclusive aquellas compo- nentes menores que la tensión umbral del diodo, que cuyo caso no ocurre en un rectificador ordinario [11], está conformado por un amplificador operacional MC1741CP y un diodo de propósito general, cuyo esquema se muestra en la Fig. 5. El comparador analiza si la señal rectificada corresponde a la de la voz o a la del ruido comparándola con el voltaje umbral de la voz, generando a su salida un voltaje continuo.

Etapa oscilador, el cual será activado por el voltaje continuo proporcionado por el comparador de la etapa circuito discriminador, generando el oscilador una señal sinusoidal pura que precisamente es la que será utilizada para ensordecer y corregir la realimentación auditiva distorsionada de la persona tartamuda. Esta etapa es conformada por un circuito sintonizador y un comparador de tensión Fig. 6 . La señal de salida se caracteriza por estar comprendida dentro del rango de frecuencia audible, $465 \mathrm{~Hz}$ a $2 \mathrm{KHz}$. [12], y cuya amplitud se mantiene constante durante todo ese intervalo, además tiene incorporado un circuito visual que indica la presencia de voz mediante el encendido de un diodo led. Esta etapa permite regular solo la frecuencia del sonido más no la intensidad.

Etapa amplificador de salida proporciona una intensidad que va desde $0 \mathrm{~dB}$. a $35 \mathrm{~dB}$ por audífono, a través del ajuste del valor del potenciómetro. Esta etapa la conforma un amplificador operacional LF351N que provee una adaptación adecuada de impedancia a los auriculares, y un selector de volumen definido por le potenciómetro P3, ver Fig. 7.

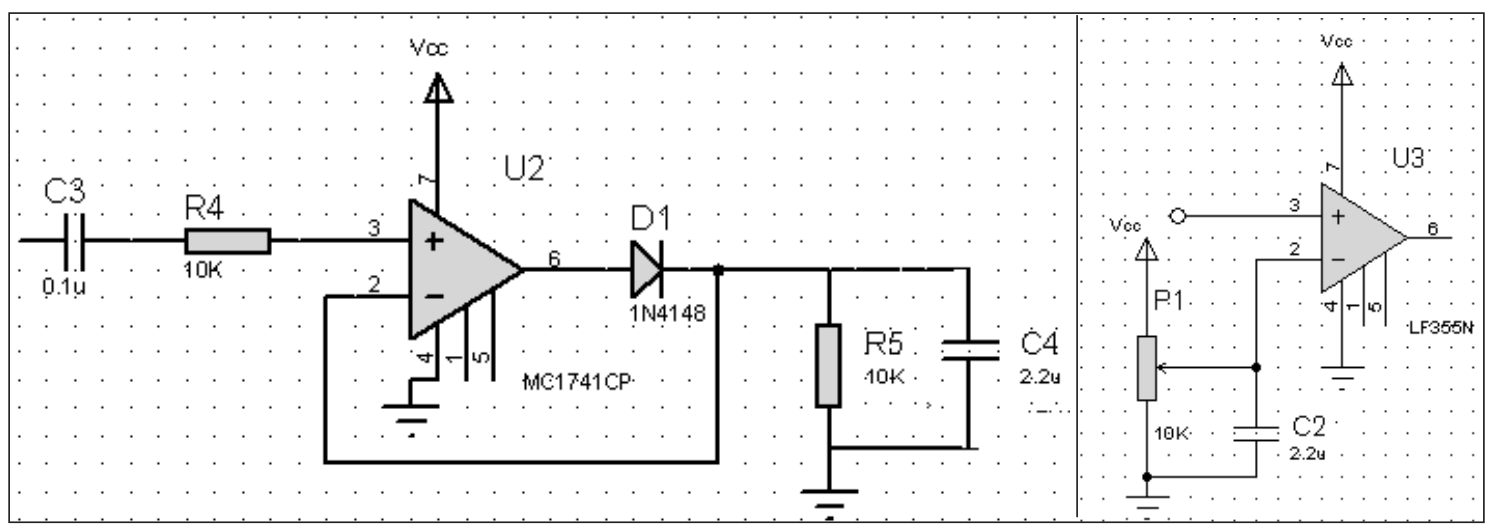

Fig. 5: Etapa Discriminador. 


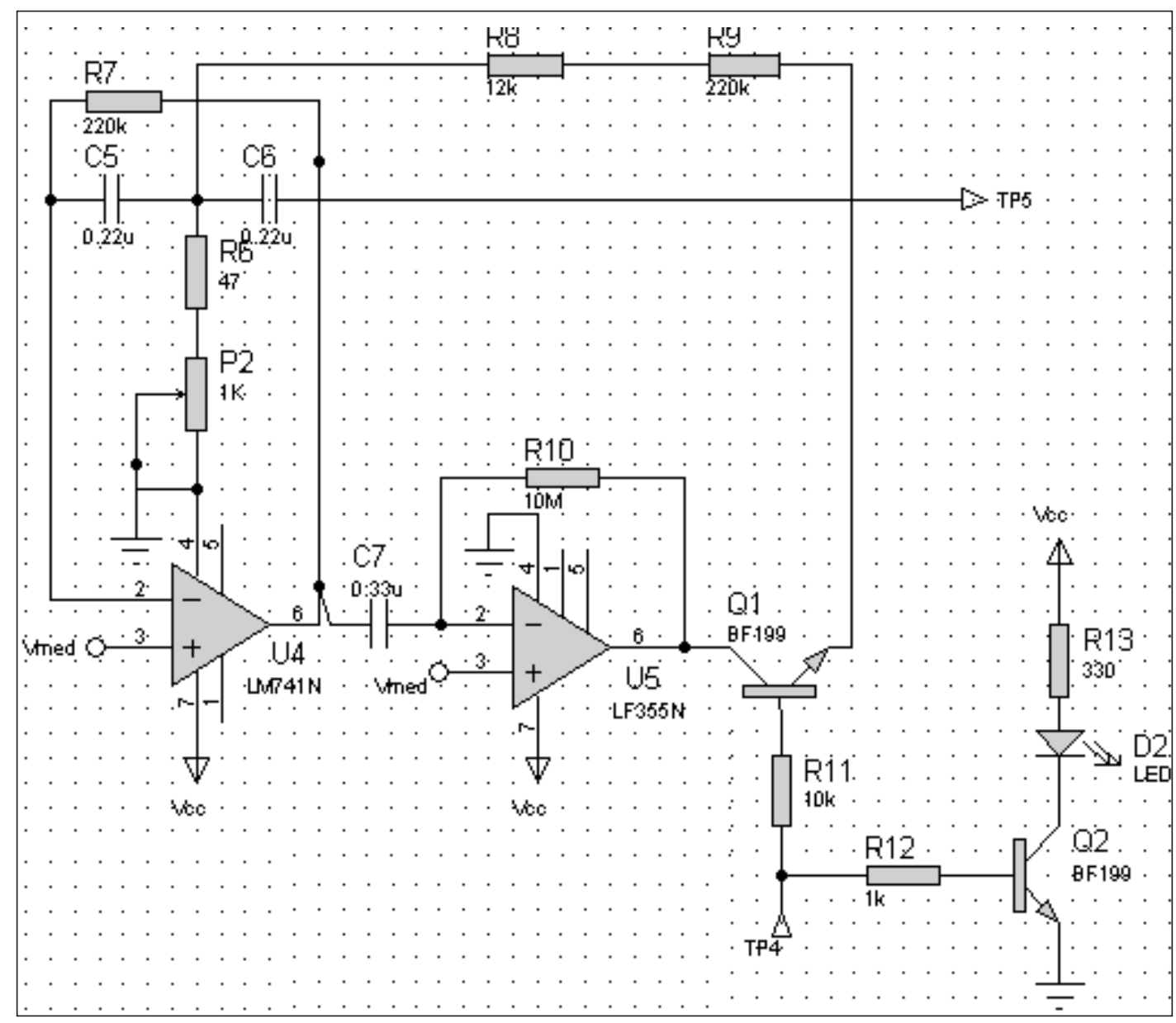

Fig. 6: Etapa Oscilador.

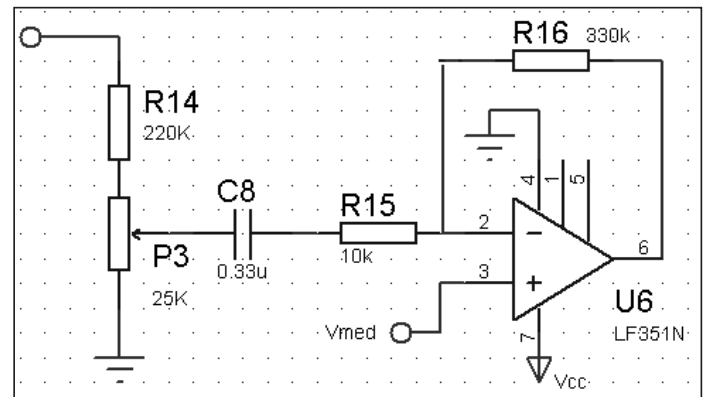

Fig. 7: Etapa Amplificador de Salida.

Etapa auricular tienen la función de convertir nuevamente la señal eléctrica a señal acústica y llevarla desde el dispositivo hasta los oídos del paciente. Los auriculares se interconectaron en seriados con una resistencia R17 para obtener una impedancia de $32 \&$ !., con la finalidad de obtener la máxima transferencia de potencia, ver Fig. 8

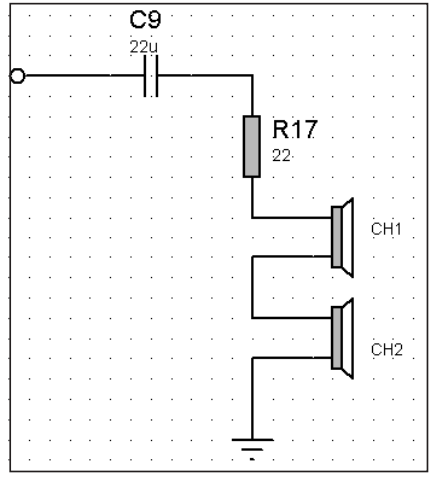

Fig. 8: Etapa Auriculares.

\section{RESULTADOS Y DISCUSIÓN}

Los siguientes gráficos se presenta las formas de onda para cada etapa que se obtuvo en el desarrollo del presente trabajo. Finalmente se muestra el desarrollo completo de dicho dispositivo 


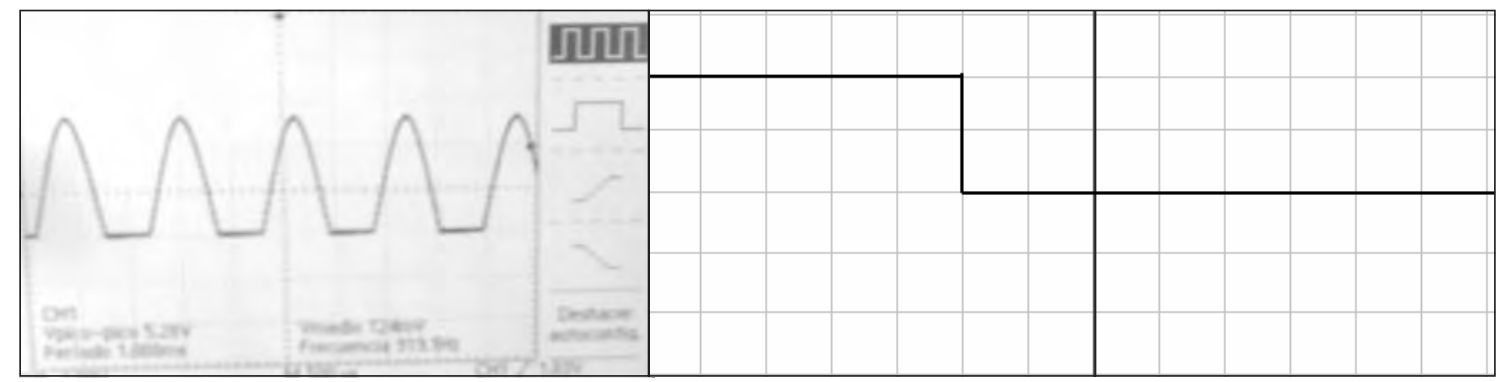

Fig. 9: Formas de Onda de la Etapa Discriminador.

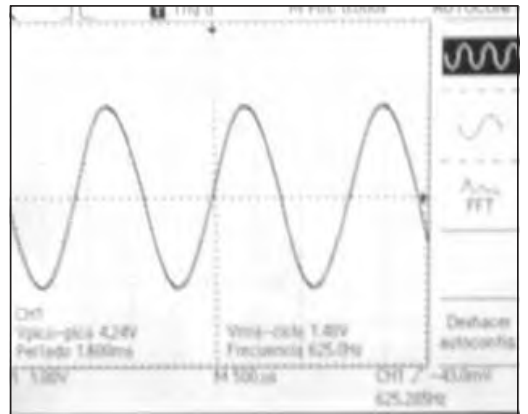

Fig. 10: Formas de Onda de la Etapa Oscilador.

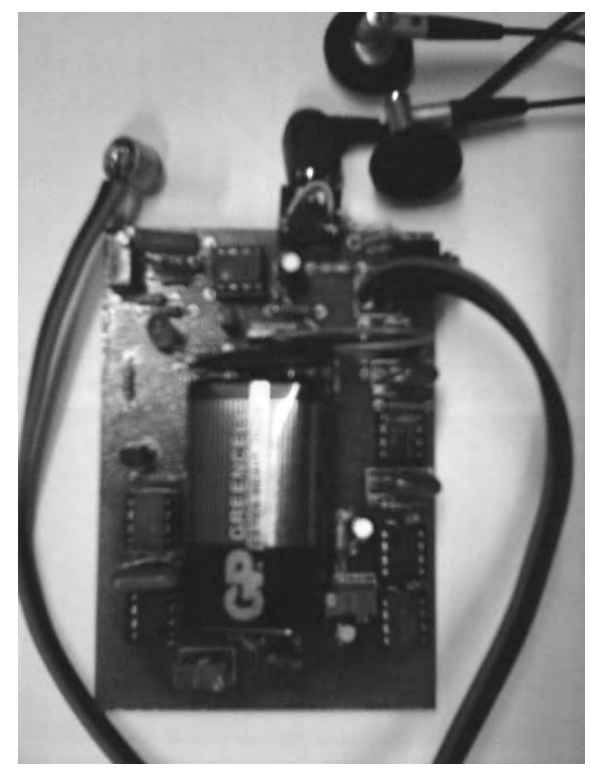

Fig. 11: Dispositivo Desarrollado.

El diseño y desarrollado de estos dispositivos contribuyen al mejoramiento de la fluidez del habla de las personas que sufren el síndrome de la tartamudez mediante la corrección de la realimentación auditiva distorsionada que presentan en dichas personas, según la bibliografía consultada. Sin embargo, el dispositivo desarrollado en el presente artículo se encuentra en la fase de prueba, lo cual implica el desarrollo de un protocolo de pruebas conjuntamente con la parte médica especializada. Así como, para la selección de pacientes, y su respectivo seguimiento para la comprobación de los beneficios hacia el paciente.

El tratamiento de la tartamudez está a cargo de un Fonoaudiólogo, que se encarga de un estudio riguroso, como el entorno del tartamudo ya sea familiar y social, métodos de respiración, etc. Este tratamiento es complementado con dispositivos electrónicos como audiómetros siendo no muy accesibles estos en nuestras población. Por lo que el dispositivo enmascarador de voz señalado en este trabajo es una herramienta muy útil para su tratamiento. Es así que este dispositivo está en su etapa de prototipo, está siendo evaluado por el especialista y el paciente, cuyas mejoras deben ser resueltas en un periodo de 4-5 meses.

\section{CONCLUSIONES}

El dispositivo ha sido construido utilizando únicamente circuitos analógicos (amplificadores operacionales) sin necesidad de ningún sistema digital con lo cual demuestra que aun sigue siendo vigente en muchas aplicaciones en un mundo con tendencia hacia la digitalización. Con los amplificadores operacionales alimentados con una fuente única (batería de $9 \mathrm{~V})$ permitió reducir aún más el tamaño del dispositivo así como un ahorro en el consumo de energía satisfaciendo su calidad de portabilidad.

Para reducir la probabilidad de captar ruidos proveniente de distintas direcciones se hace necesario el uso de micrófonos unidireccionales 
en vez de los omnidireccionales utilizados en este primer prototipo.

La intensidad y la frecuencia del sonido generado por el dispositivo se ha diseñado de tal modo que se encuentre dentro del nivel auditivo admisible, y que se ajuste a las características auditivas de cada persona (tono agradable), respectivamente.

Se ha diseñado y desarrollado un dispositivo electrónico portátil que contribuirá el mejoramiento de la fluidez del habla de las personas que sufren el síndrome de la tartamudez.

\section{REFERENCIAS BIBLIOGRÁFICAS}

[1] Teresa Tovar, Patricia Fernández. Estudio de Consulta Nacional sobre Discapacidad (CONADIS). Año 2003. Lima-Perú

[2] Pablo Félix Castañeda. UNMSM. Aparato Fonador y las partes que intervienen en el proceso del Habla. 5 de Mayo de 2005.Lima-Peru

[3] Ingrid Creus, Juan Romeu. Tratamiento Sicológico de la Disfemia. 12 de Noviembre de 2007. Barcelona

[4] Grupo de Ayuda a Tartamudos de Asturias. Van Riper, Entrevista. at http://personales.ya.com/fernian/gatastur/ Entrevista.pdf. España

[5] Calogero Bruscianelli. Universidad Simón Bolívar. Grupo de Procesamiento de Señales. Fisiología del sistema auditivo. 9 de Junio de 2005. Venezuela

[6] Wikipedia. Aparato Fonador at http://es.wikipedia.org/wiki/Aparato_fonador. 4de Agosto de 2008. España.

[7] B Domínguez Aurrecoehea. Trastornos del lenguaje en el niño. Articulo de ediciones Doyma.

[8] Van Riper, Ch. The Treatment of Stuttering

[9] Ingrid Creus. Tratamientos Clásicos a la Disfemia. 12 de Noviembre de 2007. Barcelona.

[10] Wikipedia. Microfono at http://es.wikipedia.org/wiki/Micrófono. 20 de Julio de 2008. España.

[11] R. F. Coughlin, F. F. Driscoll. Amplificadores Operacionales y circuitos integrados lineales. 5ta Ed. Prentice Hall. 1999.

[12] Wikipedia. Audiofrecuencia at http://es.wikipedia.org/wiki/Audiofrecuencia. 6 de Septiembre de 2007. España

E-mail: lyabar_gts@hotmail.com 